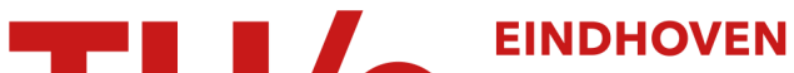 \\ UNIVERSITY OF \\ TECHNOLOGY
}

\section{Degenerate atom-molecule mixture in a cold Fermi gas}

Citation for published version (APA):

Kokkelmans, S. J. J. M. F., Shlyapnikov, G. V., \& Salomon, C. (2004). Degenerate atom-molecule mixture in a cold Fermi gas. Physical Review A : Atomic, Molecular and Optical Physics, 69(3), 31602-1/4. [31602]. https://doi.org/10.1103/PhysRevA.69.031602

DOI:

10.1103/PhysRevA.69.031602

Document status and date:

Published: 01/01/2004

\section{Document Version:}

Publisher's PDF, also known as Version of Record (includes final page, issue and volume numbers)

\section{Please check the document version of this publication:}

- A submitted manuscript is the version of the article upon submission and before peer-review. There can be important differences between the submitted version and the official published version of record. People interested in the research are advised to contact the author for the final version of the publication, or visit the $\mathrm{DOI}$ to the publisher's website.

- The final author version and the galley proof are versions of the publication after peer review.

- The final published version features the final layout of the paper including the volume, issue and page numbers.

Link to publication

\section{General rights}

Copyright and moral rights for the publications made accessible in the public portal are retained by the authors and/or other copyright owners and it is a condition of accessing publications that users recognise and abide by the legal requirements associated with these rights.

- Users may download and print one copy of any publication from the public portal for the purpose of private study or research.

- You may not further distribute the material or use it for any profit-making activity or commercial gain

- You may freely distribute the URL identifying the publication in the public portal.

If the publication is distributed under the terms of Article 25fa of the Dutch Copyright Act, indicated by the "Taverne" license above, please follow below link for the End User Agreement:

www.tue.nl/taverne

Take down policy

If you believe that this document breaches copyright please contact us at:

openaccess@tue.nl

providing details and we will investigate your claim. 


\title{
Degenerate atom-molecule mixture in a cold Fermi gas
}

\author{
S. J. J. M. F. Kokkelmans, ${ }^{1}$ G. V. Shlyapnikov, ${ }^{1,2,3, *}$ and C. Salomon ${ }^{1}$ \\ ${ }^{1}$ Laboratoire Kastler Brossel, Ecole Normale Supérieure, 24 rue Lhomond, 75231 Paris 05, France \\ ${ }^{2}$ FOM Institute AMOLF, Kruislaan 407, 1098 SJ Amsterdam, The Netherlands \\ ${ }^{3}$ Russian Research Center Kurchatov Institute, Kurchatov Square, 123182 Moscow, Russia
}

(Received 15 October 2003; published 23 March 2004)

\begin{abstract}
We show that the atom-molecule mixture formed in a degenerate atomic Fermi gas with interspecies repulsion near a Feshbach resonance constitutes a peculiar system where the atomic component is almost nondegenerate but quantum degeneracy of molecules is important. We develop a thermodynamic approach for studying this mixture, explain experimental observations, and predict optimal conditions for achieving molecular Bose-Einstein condensation.
\end{abstract}

DOI: 10.1103/PhysRevA.69.031602

Interactions between particles play a crucial role in the behavior of degenerate quantum gases. For instance, the sign of the effective mean-field interaction determines the stability of a large Bose-Einstein condensate (BEC), and the shape of such a condensate in a trap can be significantly altered from its ideal-gas form [1]. In degenerate Fermi gases the effects of mean-field interactions are usually less pronounced in the size and shape of the trapped cloud, and these quantities are mostly determined by Fermi statistics. The strength of the interactions, however, can be strongly increased by making use of a Feshbach resonance [2,3], and then the situation changes.

Recent experiments present two types of measurement of the interaction energy in a degenerate two-component Fermi gas near a Feshbach resonance [4-7]. At JILA [5] and at MIT [7] the mean-field energy was found from the frequency shift of a rf transition for one of the atomic states. The results are consistent with the magnetic-field dependence of the scattering length $a$, the energy being positive for $a>0$ and negative for $a<0$. In the Duke [4] and ENS [6] experiments with ${ }^{6} \mathrm{Li}$, the results are quite different. The interaction energy was obtained from the measurement of the size of an expanding cloud released from the trap. A constant ratio of the interaction to Fermi energy, $E_{\text {int }} / E_{F} \approx-0.3$, was found around resonance, irrespective of the sign of $a[4,6]$. It was explained in Ref. [4] by claiming a universal behavior in this strongly interacting regime [8]. The ENS studies in a wide range of magnetic fields [6] found that $E_{\text {int }}$ changes to a large positive value when $a$ is tuned positive, but only at a field strongly shifted from resonance.

In contrast to the JILA [5] and MIT [7] studies providing a direct measurement of the mean-field interaction energy, the Duke [4] and ENS [6] experiments measure the influence of the interactions on the gas pressure. An interpretation of the ENS experiment involves the creation of weakly bound molecules via three-body recombination at a positive $a$ [6].

\footnotetext{
*Present address: Laboratoire Physique Théorique et Modèles Statistique, Université Paris Sud, Bât. 100, 91405 Orsay Cedex, France, and van der Waals-Zeeman Institute, University of Amsterdam, 1018 XE Amsterdam, The Netherlands.
}

PACS number(s): 03.75.Ss, 05.30.Jp, 05.20.Dd, 32.80.Pj

Far from resonance, the binding energy of the produced molecules and, hence, their kinetic energy are larger than the trap depth and the molecules escape from the trap. The interaction energy is then determined by the repulsive interaction between atoms and is positive [6]. Close to resonance, the three-body recombination is efficient [9] and the molecules remain trapped as their binding energy $\epsilon_{B}$ is smaller than the trap depth $[6,9]$. They come to equilibrium with the atoms, reducing the pressure in the system.

Away from resonances, the interaction strength is proportional to $a$, and is given by $g=4 \pi \hbar^{2} a / M$, with $M$ the atom mass. Close to resonance this relation is not valid, as the value of $|a|$ diverges to infinity and the scattering process strongly depends on the collision energy. For Boltzmann gases, already in the 1930s, Beth and Uhlenbeck [10] calculated the second virial coefficient by including both the scattering and bound states for the relative motion of pairs of atoms [11]. A small interaction-induced change of the pressure in this approach is negative on both sides of the resonance $[12,13]$.

However, current experiments are not in the Boltzmann regime. In this paper we show that the atom-molecule mixture formed in a cold atomic Fermi gas constitutes a peculiar system in which the atomic component is almost nondegenerate, whereas quantum degeneracy of the molecules can be very important. This behavior originates from a decrease of the atomic fraction with temperature. It is present even if the initial Fermi gas is strongly degenerate in which case almost all atoms are converted into molecules. We develop a thermodynamic approach for studying this mixture, predict optimal conditions for achieving molecular BEC, and properly describe the interaction effects as observed at ENS [6].

We assume that fermionic atoms are in equilibrium with weakly bound (bosonic) molecules formed in the recombination process. The molecules are treated as point bosons. Atom-molecule and molecule-molecule interactions are omitted at first, and will be discussed in a later stage. For a large scattering length $a>0$, the binding energy of the weakly bound molecules is $\epsilon_{B}=\hbar^{2} /\left(M a^{2}\right)$, and their size is roughly given by $a / 2$. For treating them as point bosons, this size should be smaller than the mean interparticle separation. This requires the inequality $n(a / 2)^{3}<1$, which at densities 
$n \approx 10^{13} \mathrm{~cm}^{-3}$ is satisfied for $a<18000 a_{0}$, and excludes a narrow vicinity of the Feshbach resonance.

The presence of molecules reduces the number of particles in the atomic component and to an essential extent lifts its quantum degeneracy. The molecular chemical potential is negative in the absence of atom-molecule and moleculemolecule mean field, and thermal equilibrium between atoms and molecules requires a negative chemical potential for the atoms. We thus assume a priori that the occupation numbers of the states of atoms are small. This proves to be the case at any temperature, except for very low $T$ where the atomic fraction is negligible. Under these conditions we omit pairing correlations between the atoms, which are important for describing a crossover from the BCS to BEC regime [14-17] and can be expected even in the nonsuperfluid state.

Assuming equal densities of the atomic components, labeled as $\uparrow$ and $\downarrow$, their chemical potentials are $\mu_{\uparrow}=\mu_{\downarrow}=\mu$, where $\mu$ is the chemical potential of the system as a whole. The molecular chemical potential is $\mu_{m}=-\epsilon_{B}+\tilde{\mu}_{m}$, with $\tilde{\mu}_{m}$ $\leqslant 0$ being the chemical potential of an ideal gas of bosons with the mass $2 M$. The condition of thermal equilibrium $\mu_{\uparrow}+\mu_{\downarrow}=\mu_{m}$ then reads

$$
2 \mu=-\epsilon_{B}+\tilde{\mu}_{m} .
$$

From Eq. (1) we will obtain the number of molecules $N_{m}$ and the number of atoms $N_{a}$ for given temperature $T$ and total number of atomic particles $N=N_{a}+2 N_{m}$. This requires us to obtain the expression for the occupation numbers of the atoms and the dependence of $\mu$ on $N_{a}$.

The main difficulty with constructing a thermodynamic approach for the degenerate molecule-atom mixture is related to the resonance momentum-dependent character of the atom-atom interactions. This difficulty is circumvented for small occupation numbers of the atoms. Then, even at resonance, the interaction energy is equal to the mean value of the interaction potential for a given relative momentum of a colliding pair, averaged over the momentum distribution. In this respect, the interaction problem becomes similar to the calculation of the total energy of a heavy impurity as caused by its interactions with the surrounding electrons in a metal [19]. This approach leads to a relation between the collisioninduced shift of the energy levels of particles in a large spherical box, and the scattering phase shift. Adding the integration over the states of the center-of-mass motion for pairs of atoms, we find that the total energy of interatomic interaction is equal to $\sum_{\mathbf{k k}^{\prime}} g_{\mathbf{k k}^{\prime}} \nu_{\uparrow}(k, \mu, T) \nu_{\downarrow}\left(k^{\prime}, \mu, T\right) / V$, where $\nu_{\uparrow}$ and $\nu_{\downarrow}$ are occupation numbers of single-particle momentum states, and $V$ is the volume (cf. Ref. [19]). The momentum-dependent coupling constant is given by

$$
g_{\mathbf{k k}^{\prime}}=-\frac{4 \pi \hbar^{2}}{M} \frac{\delta\left(\left|\mathbf{k}-\mathbf{k}^{\prime}\right| / 2\right)}{\left|\mathbf{k}-\mathbf{k}^{\prime}\right| / 2} .
$$

The phase shift $\delta$ is expressed through the relative momentum $q=\left|\mathbf{k}-\mathbf{k}^{\prime}\right| / 2$ and the scattering length $a$ as $\delta$ $=-\arctan (q a)$. In the limit of $q|a| \ll 1$, Eq. (2) transforms into the ordinary coupling constant $g=4 \pi \hbar^{2} a / M$.
As we have $\nu_{\uparrow}(k, \mu, T)=\nu_{\downarrow}(k, \mu, T) \equiv \nu_{k}$, the total energy of the atomic component and the number of particles in this component can be written in the form

$$
E_{a}=\sum_{\mathbf{k}} \frac{\hbar^{2} k^{2}}{M} \nu_{k}+\sum_{\mathbf{k k}^{\prime}} \frac{g_{\mathbf{k k}^{\prime}}}{V} \nu_{k} \nu_{k^{\prime}}, \quad N_{a}=2 \sum_{\mathbf{k}} \nu_{k} .
$$

In our mean-field approach, the entropy of the atoms is given by the usual combinatorial expression [18]

$$
S_{a}=-2 \sum_{\mathbf{k}}\left[\nu_{k} \ln \nu_{k}+\left(1-\nu_{k}\right) \ln \left(1-\nu_{k}\right)\right]
$$

Equations (3) and (4) immediately lead to an expression for the atomic grand potential $\Omega_{a}=E_{a}-T S_{a}-\mu N_{a}$. Then, using the relation $N_{a}=-\left(\partial \Omega_{a} / \partial \mu\right)_{T, V}$, we obtain for the occupation numbers of atoms

$$
\nu_{k}=\left[\exp \left\{\left(\epsilon_{k}-\mu\right) / T\right\}+1\right]^{-1},
$$

where $\epsilon_{k}=\hbar^{2} k^{2} / 2 M+U_{k}$, and $U_{k}=\sum_{\mathbf{k}^{\prime}} g_{\mathbf{k k}^{\prime}} \nu_{k^{\prime}} / V$ is the mean field acting on the atom with momentum k. Accordingly, the expression for the grand potential and pressure of the atomic component reads

$$
\Omega_{a}=-P_{a} V=\sum_{\mathbf{k}}\left[2 T \ln \left(1-\nu_{k}\right)-U_{k} \nu_{k}\right] .
$$

This set of equations is completed by the relation between the density of bosonic molecules and their chemical potential. In the absence of molecular BEC we have

$$
n_{m}=\left(\sqrt{2} / \Lambda_{T}\right)^{3 / 2} g_{3 / 2}\left[\exp \left(\tilde{\mu}_{m} / T\right)\right]
$$

where $g_{\alpha}(x)=\sum_{j=1}^{\infty} x^{j} / j^{\alpha}$, and $\Lambda_{T}=\left(2 \pi \hbar^{2} / M T\right)^{1 / 2}$ is the thermal de Broglie wavelength for the atoms. For $n_{m} \Lambda_{T}^{3}>7.38$ the molecular fraction becomes Bose condensed, and we have $\tilde{\mu}=0$ and $\mu=-\epsilon_{B} / 2$. Similarly, the energy, entropy, and grand potential of the molecules are given by usual equations for an ideal Bose gas [11].

From Eqs. (1)-(7) we obtain the fraction of unbound atoms $n_{a} / n$ and the fraction of atoms bound into molecules, $2 n_{m} / n$, as universal functions of two parameters: $T / \epsilon_{B}$ and $n \Lambda_{T}^{3}$, where $n$ is the total density of atomic particles. The dependence of atomic and molecular fractions on $T / \epsilon_{B}$ for two values of $n \Lambda_{T}^{3}$ is shown in Fig. 1. The molecular fraction increases and the atomic fraction decreases with decreasing $T / \epsilon_{B}$. Occupation numbers of the atoms are always small, whereas quantum degeneracy of molecules is important. The dotted line in Fig. 1(b) indicates the onset of molecular BEC.

This mixture was realized in the ENS experiment [6], where the occupation numbers for the molecules were up to 0.3 and the molecular fraction was exceeding the atomic one. In the recent studies [20-23] almost all atoms were converted into molecules by sweeping the magnetic field across the resonance, and at ENS [20] the temperature was within a factor of 2 from molecular BEC. Remarkably, one can modify the molecular fraction and degeneracy parameter $n_{m} \Lambda_{T}^{3}$ by adiabatically tuning the atom-atom scattering length, as shown in Fig. 2. The decrease of $a$ increases the binding energy $\epsilon_{B}$ and the molecular fraction, and thus 
(a)

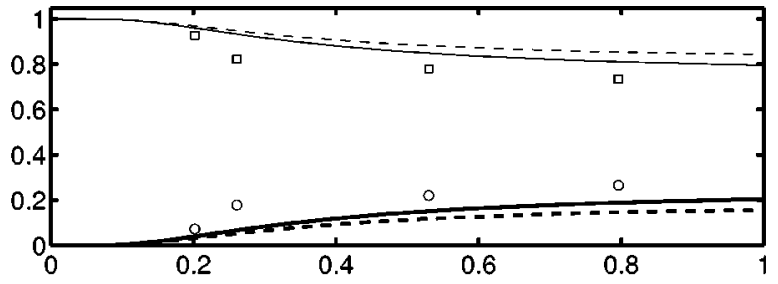

(b)

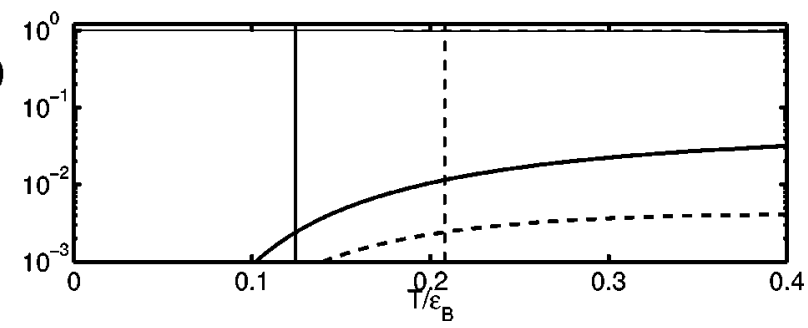

FIG. 1. Fraction of unbound atoms $n_{a} / n$ (lower curves, bold) and fraction of atoms bound into molecules, $2 n_{m} / n$ (upper curves) vs $T / \epsilon_{B}$ : (a) $n \Lambda_{T}^{3}=2.5$, squares and circles show the ENS data [20]; (b) $n \Lambda_{T}^{3}=14.8$, and the vertical lines indicate the onset of molecular BEC. Dashed curves are obtained including atom-molecule and molecule-molecule interactions.

causes heating [20]. Close to resonance, $n_{m} \Lambda_{T}^{3}$ remains almost constant and then decreases due to heating.

The atom-molecule and molecule-molecule interactions are readily included in our approach for $a \ll \Lambda_{T}$, where the corresponding coupling constants are $g_{a m}=0.9 \mathrm{~g}$ and $g_{m}$ $=0.3 \mathrm{~g}$ [26]. In this limit the interactions provide an equal shift of the chemical potential and single-particle energy $\epsilon_{k}$. For the atoms this shift is $n_{a} g / 2+n_{m} g_{a m}$, where the first term is the atom-atom contribution $U_{k}$. For the (noncondensed) molecules the shift is $n_{a} g_{a m}+2 n_{m} g_{m}$. The entropy of the mixture is given by the same expressions as in the absence of the interactions. As seen in Figs. 1 and 2, the atom-molecule and molecule-molecule interactions do not significantly modify our results. From Fig. 2 one then concludes that the conditions for achieving molecular BEC are optimal for values of $a$ as low as possible while still staying at the plateau, as at larger $a$ the interaction between the molecules can reduce the BEC transition temperature [1].

We now analyze the interaction effect observed at ENS for trapped clouds in the hydrodynamic regime [6]. The experiment was done near the Feshbach resonance located at

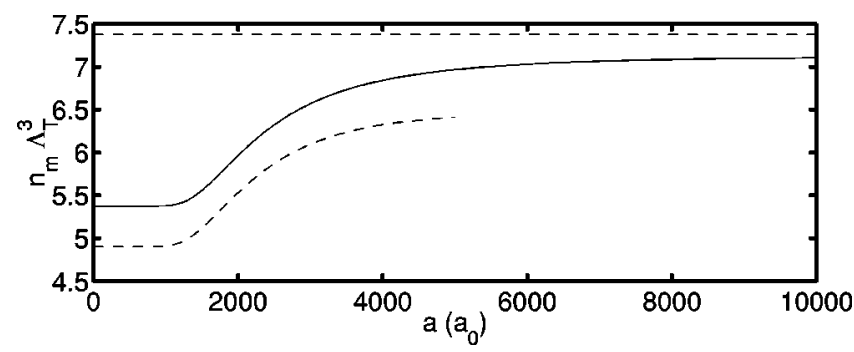

FIG. 2. Molecular degeneracy parameter $n_{m} \Lambda_{T}^{3}$ under adiabatic variation of $a$ for ${ }^{6} \mathrm{Li}$, assuming $n \Lambda_{T}^{3}=15$ close to resonance. The dashed curve is obtained including atom-molecule and moleculemolecule interactions. The horizontal dashed line shows the critical value for molecular BEC.

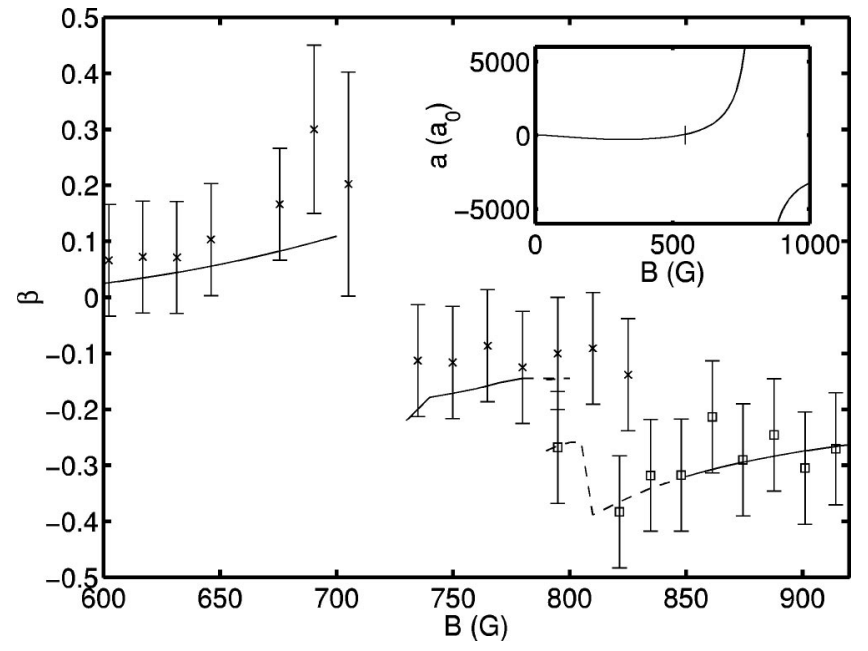

FIG. 3. Calculated (solid line) and measured [6] (squares and crosses) ratio $\beta$ of the interaction to kinetic energy (see text). The calculated line for $B>790 \mathrm{G}$ is for experimental conditions $T$ $=0.9 E_{F}=3.4 \mu \mathrm{K}$ and $n=3 \times 10^{13} \mathrm{~cm}^{-3}$. For $B<700 \mathrm{G}$ we take the averaged experimental conditions $T=1.1 E_{F}=2.4 \mu \mathrm{K}$ and $n$ $=1.3 \times 10^{13} \mathrm{~cm}^{-3}$. For $700<B<800 \mathrm{G}$, we use the local conditions (see Ref. [6]). Inset: Scattering length as a function of magnetic field.

the magnetic field $B_{0}=810 \mathrm{G}$, and the data results from two types of measurements of the size of the cloud released from the optical trap. In the first one, the magnetic field and, hence, the scattering length are kept the same as in the trap. Therefore, the cloud expands with the speed of sound $c_{s}$ $=\sqrt{(\partial P / \partial \rho)_{S}}$, where $\rho=m n$ is the mass density. The speed $c_{s}$ and, hence, the size of the expanding cloud are influenced by the presence of molecules and by the interparticle interactions.

In the second type of measurement, the magnetic field is first rapidly ramped down and the scattering length becomes almost zero on a time scale $t \sim 2 \mu$ s. This time scale is short compared to the collisional time. Therefore, the spatial distribution of the atoms remains the same as in the initial cloud, although the mean field is no longer present. At the same time, a rapid decrease of $a$ increases the binding energy of molecules $\epsilon_{B}$. However, as the time $t \lesssim \hbar / \epsilon_{B}$, they cannot adiabatically follow to a deeper bound state and dissociate into atoms which acquire kinetic energy. Thus the system expands symmetrically as an ideal gas of $N$ atoms, with the initial density profile. The momentum distribution $f_{k}$ will be a sum of the initial atomic momentum distribution and one that arises from the dissociated molecules. The latter is found assuming an abrupt change of $a$ and, hence, projecting the molecular wave function on a complete set of plane waves. This gives rise to a distribution $c(q)$ for the relative momentum $q$. The single-particle momentum distribution for the atoms produced out of molecules results then from convoluting $c\left(\left|\mathbf{k}-\mathbf{k}^{\prime}\right| / 2\right)$ with the molecular distribution function $\nu_{m}\left(\mathbf{k}+\mathbf{k}^{\prime}\right)$ by integrating over $\mathbf{k}^{\prime}$. One can establish a relation between the expansion velocity $v_{0}$ of this nonequilibrium system and the expansion velocity $c_{0}$ of an ideal equilibrium two-component atomic Fermi gas which has the same density and temperature: $4 \pi^{3} n \int_{0}^{M v_{0} / \hbar} d k k^{2} f_{k}$ 
$=\int_{0}^{M c_{0} / \hbar} d k k^{2} \widetilde{\nu}_{k}$, with $\widetilde{\nu}_{k}$ being the ideal-gas momentum distribution. Using the scaling approach [24,25], one can find that in the spherical case the velocity $c_{0}$ coincides with the expansion velocity of the hydrodynamic Fermi gas in the absence of mean-field interactions and, accordingly, is given by $c_{0}^{2}=5 P_{0} / 3 \rho$, where $P_{0}=2 E_{0} / 3 V$ is the pressure.

The relative difference between the squared size of the expanding cloud in the two described cases can be treated as the ratio of the interaction to kinetic energy and called the interaction shift. This interaction shift is then given by the relative difference between the two squared velocities: $\beta$ $=\left[c_{s}^{2}-v_{0}^{2}\right] / v_{0}^{2}$. Our results for this quantity are calculated for experimental conditions and are presented in Fig. 3. The sound velocity $c_{s}$ was obtained using the above developed approach including only atom-atom interactions. The field region where $n(a / 2)^{3}>1$ is beyond the validity of this approach and is shown by the dashed curve. In Fig. 3 we also show our previous results for fields $B>810 \mathrm{G}(a<0)$ and $B<700 \mathrm{G}\left(0<a<2000 a_{0}\right)$, where molecules are absent [6].

Our quantum-statistical approach gives a negative interaction shift on both sides of the Feshbach resonance, in good quantitative agreement with the experiment. Without molecules present, the interaction energy would jump to positive values left from resonance, as can be seen from our calculation in Ref. [6]. This demonstrates that the apparent field shift from resonance, where a sign change in the interaction energy is observed, is an indirect signature of the presence of molecules in the trap.

For high temperatures $T \gg E_{F}$ and small binding energy
$\epsilon_{B} \ll T$, we find that $\beta$ has a universal behavior and is proportional to the second virial coefficient. However, this only holds at high temperatures (cf. Ref. [13]), and at low $T$ the molecule-molecule interaction can strongly influence the result. For $T$ approaching the temperature of molecular BEC, which is $T_{c} \approx \hbar^{2} n^{2 / 3} / M \approx 0.2 E_{F}$, the atomic fraction is already small and the sound velocity $c_{s}$ is determined by the molecular cloud. For $a \ll \Lambda_{T}$ we find $c_{s}^{2}=0.4 T_{c} / M$ $+n g_{m} / 2 M$, where the second term is provided by the molecule-molecule interaction and is omitted in the high- $T$ approach. The ratio of this term to the first one is $\sim 5\left(n a^{3}\right)^{1 / 3}$. For $B=700 \mathrm{G}$ at densities of Ref. [6], it is equal to 1 and is expected to grow when approaching the resonance.

Thus, except for a narrow region where $n|a|^{3} \gg 1$, one cannot speak of a universal behavior of the shift $\beta$ on both sides of the resonance. The situation depends on possibilities of creating an equilibrium atom-molecule mixture. Moreover, at low temperatures the universality can be broken by the molecule-molecule interactions.

We are grateful to T. Bourdel, J. Cubizolles, C. Lobo, and L. Carr for stimulating discussions. This work was supported by the Dutch Foundations NWO and FOM, by INTAS, and by the Russian Foundation for Fundamental Research. S.J.J.M.F.K. acknowledges a Marie Curie Grant No. MCFI2002-00968 from the EU Laboratoire Kastler Brossel, which is a Unité de Recherche de l'Ecole Normale Supérieure et de l'Université Paris 6, associée au CNRS.
[1] F. Dalfovo, S. Giorgini, L. P. Pitaevskii, and S. Stringari, Rev. Mod. Phys. 71, 463 (1999).

[2] H. Feshbach, Ann. Phys. (N.Y.) 5, 357 (1958); 19, 287 (1962).

[3] E. Tiesinga et al., Phys. Rev. A 46, R1167 (1992).

[4] K. M. O'Hara et al., Science 298, 2179 (2002); M. E. Gehm et al., Phys. Rev. A 68, 011401(R) (2003).

[5] C. A. Regal and D. S. Jin, Phys. Rev. Lett. 90, 230404 (2003).

[6] T. Bourdel et al., Phys. Rev. Lett. 91, 020402 (2003).

[7] S. Gupta et al., Science 300, 1723 (2003).

[8] H. Heiselberg, Phys. Rev. A 63, 043606 (2001).

[9] D. S. Petrov, Phys. Rev. A 67, 010703 (2003).

[10] E. Beth and G. E. Uhlenbeck, Physica (Utrecht) 4, 915 (1937).

[11] E. M. Lifshitz and L. P. Pitaevskii, Statistical Physics, Part 1 (Pergamon Press, Oxford, 1980).

[12] L. P. Pitaevskii and S. Stringari (private communication).

[13] T. L. Ho and E. J. Mueller, e-print cond-mat/0306187; T. L. Ho, e-print cond-mat/0309109.

[14] P. Nozières and S. Schmitt-Rink, J. Low Temp. Phys. 59, 195 (1985).

[15] M. Randeria, in Bose-Einstein Condensation, edited by A.
Griffin, D. W. Snoke, and S. Stringari (Cambridge University Press, Cambridge, 1995).

[16] Y. Ohashi and A. Griffin, Phys. Rev. Lett. 89, 130402 (2002).

[17] J. N. Milstein, S. J. J. M. F. Kokkelmans, and M. J. Holland, Phys. Rev. A 66, 043604 (2002).

[18] E. M. Lifshitz and L. P. Pitaevskii, Statistical Physics, Part 2 (Pergamon Press, Oxford, 1980).

[19] G. D. Mahan, Many-Particle Physics (Plenum Press, New York, 1990); F. G. Fumi, Philos. Mag. 46, 1007 (1955).

[20] J. Cubizolles et al., Phys. Rev. Lett. 91, 240401 (2003).

[21] S. Jochim et al., Phys. Rev. Lett. 91, 240402 (2003).

[22] K. E. Strecker, G. B. Partridge, and R. G. Hulet, Phys. Rev. Lett. 91, 080406 (2003).

[23] C. A. Regal, M. Greiner, and D. S. Jin, e-print cond-mat/ 0308606.

[24] Y. Kagan, E. L. Surkov, and G. V. Shlyapnikov, Phys. Rev. A 55, R18 (1997).

[25] C. Menotti, P. Pedri, and S. Stringari, Phys. Rev. Lett. 89, 250402 (2002).

[26] D. S. Petrov, C. Salomon, and G. V. Shlyapnikov, e-print cond-mat/0309010. 